\title{
Targeted gold nanoparticles enable molecular CT imaging of cancer: an in vivo study
}

This article was published in the following Dove Press journal:

International Journal of Nanomedicine

10 November 2011

Number of times this article has been viewed

\section{Tobi Reuveni ${ }^{1}$ \\ Menachem Motiei' \\ Zimam Romman ${ }^{2}$ \\ Aron Popovtzer ${ }^{3}$ \\ Rachela Popovtzer' \\ 'Faculty of Engineering and the Institute of Nanotechnology and Advanced Materials, Bar-ilan University, \\ Ramat Gan, ${ }^{2} \mathrm{GE}$ HealthCare, \\ Tirat Hacarmel, ${ }^{3}$ Department of Otorhinolaryngology, Head and Neck Surgery and Onology, Davidoff Center, Rabin Medical Center, Beilinson \\ Campus, Petah Tiqwa, Israel}

Correspondence: Rachela Popovtzer Faculty of Engineering, Bar-ilan University, Ramat Gan 52900, Israel

$\mathrm{Tel}+97235317509$

Fax +9723738405

Email rachela.popovtzer@biu.ac.il
Abstract: In recent years, advances in molecular biology and cancer research have led to the identification of sensitive and specific biomarkers that associate with various types of cancer. However, in vivo cancer detection methods with computed tomography, based on tracing and detection of these molecular cancer markers, are unavailable today. This paper demonstrates in vivo the feasibility of cancer diagnosis based on molecular markers rather than on anatomical structures, using clinical computed tomography. Anti-epidermal growth factor receptor conjugated gold nanoparticles $(30 \mathrm{~nm})$ were intravenously injected into nude mice implanted with human squamous cell carcinoma head and neck cancer. The results clearly demonstrate that a small tumor, which is currently undetectable through anatomical computed tomography, is enhanced and becomes clearly visible by the molecularly-targeted gold nanoparticles. It is further shown that active tumor targeting is more efficient and specific than passive targeting. This noninvasive and nonionizing molecular cancer imaging tool can facilitate early cancer detection and can provide researchers with a new technique to investigate in vivo the expression and activity of cancer-related biomarkers and molecular processes.

Keywords: functional computed tomography, molecular imaging, gold nanoparticles, biologically targeted in vivo imaging, contrast agents

\section{Background}

The last decade has brought about major advances in cancer treatment, including the development of highly conformal radiation treatments and robotic and endoscopic surgery. These techniques rely on accurate target delineation and visualization of tumor targets, and require accuracy on the millimeter scale. Therefore, a key priority in cancer research involves the development of highly sensitive and specific imaging techniques that could vastly improve treatment capabilities through early detection of millimeter-sized tumors.

Computed tomography (CT) is among the most convenient imaging/diagnostic tools in hospitals today in terms of availability, efficiency, and cost. Undisputedly, this is one of the leading technologies applied in overall cancer management. As a diagnostic tool, CT provides valuable anatomical information regarding tumor location, size, and spread. However, the sensitivity of CT is limited in the detection of subcentimeter lesions and its specificity is relatively low, resulting in $\sim 15 \%$ false positive results (noncancerous findings that are interpreted as tumors). ${ }^{1}$ Therefore, improving current CT capabilities is critical to cancer detection. This aim can be achieved by expanding the role of CT beyond its present structural imaging capabilities and providing it with functional and molecular-based imaging capacities as well. 
The development of contrast agents for molecular imaging is an emerging field that integrates knowledge in chemistry, engineering, and molecular biology to gain information on biological processes and to identify diseases based on molecular markers, which appear before their clinical symptoms. ${ }^{2,3}$

Recently, various types of nanoprobes have been developed as blood pool CT contrast agents, such as gold nanoprobes and nanotags, ${ }^{48}$ iodine-based emulsions, ${ }^{9}$ and tantalum oxide nanoparticles. ${ }^{10}$ Blood pool contrast agents are mainly valuable for early detection of structural and functional abnormalities such as those caused by thrombi and atherosclerosis lesions in the peripheral vessels. ${ }^{7}$ Indeed, these agents have demonstrated strong X-ray attenuation that allow sharp blood vessel delineation, in addition to their prolonged circulation time. Moreover, blood pool contrast agents are also capable of cancer detection given the leaky nature of tumor vasculature, which allows for nanoparticle penetration and accumulation in the tumor. ${ }^{11-13}$ This passively targeted mechanism is caused by the enhanced permeability and retention effect. ${ }^{14,15}$

Cancer detection with actively targeted CT contrast agents takes advantage of the overexpression of specific surface receptors on cancer cells and of the ability to create nanoparticles that can specifically home to these receptors. ${ }^{16}$ An important advantage of the active targeting approach is the specificity of the findings; however, since this approach is based on the existence and degree of overexpression of specific tissue biomarkers, it can be applicable only under particular biological conditions. Hainfeld et al recently showed that gold nanoparticles (GNPs) can enhance the visibility of millimeter-sized human breast tumors in mice and that active tumor targeting (with anti-Her2 antibodies) is more efficient than passive targeting. ${ }^{17}$ Chanda et al demonstrated enhanced CT attenuation of Bombesin functionalized GNPs that selectively targeted cancer receptor sites. ${ }^{18}$ However, in vivo CT cancer detection through application of high atomic number contrast agents remains challenging due to the large amount of gold that must be delivered and accumulated on the tumor in order to induce sufficient signal to noise ratio in CT. This key factor - the total amount of gold per voxel (three-dimensional pixel) - is determined mainly by (1) nanoparticle size and their cellular labeling efficiency, and (2) the number of overexpressed receptors on the cancer cell's surface.

In this study, based on substantial prior research that investigated the delivery of nanoparticles through the tumor vasculature, $30 \mathrm{~nm}$ GNPs were utilized in order to achieve a large amount of gold per voxel. Previous studies demonstrated that $30 \mathrm{~nm}$ polyethylene glycol (PEG)ylated GNPs traverse the systemic circulation, remain undetected by the mononuclear phagocyte system, permeate through transendothelial pores in tumor blood vessels, and are able to circulate in the blood long enough to reach their target. ${ }^{19-21}$

The head and neck squamous cell carcinoma (SCC) was selected as a model system to study, since such tumors express an extremely high level of EGFR. ${ }^{22}$ In addition, a direct correlation exists between the degree of EGFR overexpression and tumor aggressiveness. ${ }^{23}$ The anti-EGFR antibody is known to be specific to head and neck SCC tumors and it is well demonstrated as nontoxic in therapeutic concentrations in humans. ${ }^{24}$ In this study, the ability of anti-EGFR-targeted GNPs to form a concentrated assembly exclusively on the SCC cancer cells in tumor-bearing mice was investigated. It is demonstrated that the strong selective $\mathrm{X}$-ray attenuation by gold nanoprobes assembly, which is distinct from the attenuation of other cell types and tissues, transforms the targeted tumor into highly distinct and easy to diagnose features in CT imaging. This study also demonstrates that active tumor targeting was more efficient than passive targeting (at specific time points), possibly because of its elevated tumor uptake and retention forces. ${ }^{25}$

\section{Methods}

\section{Preparation of particles}

As targeted contrast agents, $30 \mathrm{~nm}$ GNPs were prepared using sodium citrate, according to the methodology described by Enüstun and Turkevich. ${ }^{26}$ Particle size, shape, and uniformity were measured using transmission electron microscopy and proved to be $30 \mathrm{~nm}$ diameter spheres with narrow size distribution (10\%), as shown in Figure 1. A protective layer of PEG was absorbed on the surface of the GNPs in order to reduce nonspecific interactions and to prolong circulation time of the nanoparticles in the blood stream. ${ }^{27}$ The PEG layer consisted of a mixture of thiol-polyethylene-glycol (mPEG-SH) ( 85\%, MW $\sim 5 \mathrm{kDa})$ and a heterofunctional thiol-PEG-acid (SH-PEG-COOH) ( 15\%, MW $\sim 3.4 \mathrm{kDa})$ (Creative PEGWorks, Winston Salem, NC).

For cancer cell targeting, the heterofunctional PEG was covalently conjugated to an anti-EGFR monoclonal antibody (Erbitux $^{\circledR}$, Merck KGaA, Darmstadt, Germany), and as a negative control to an anti-rabbit immunoglobulin $\mathrm{G}$ ( $\operatorname{IgG})$ antibody (Jackson ImmunoResearch Laboratories, Inc, West Grove, PA), using 1-ethyl-3-(3-(dimethylaminopropyl)carbodiimide and $\mathrm{N}$-hydroxysulfosuccinimide (Thermo Fisher Scientific, Inc, Rockford, IL). ${ }^{28,29}$ The antibodyconjugated GNPs were stable for up to 3 months, confirmed 

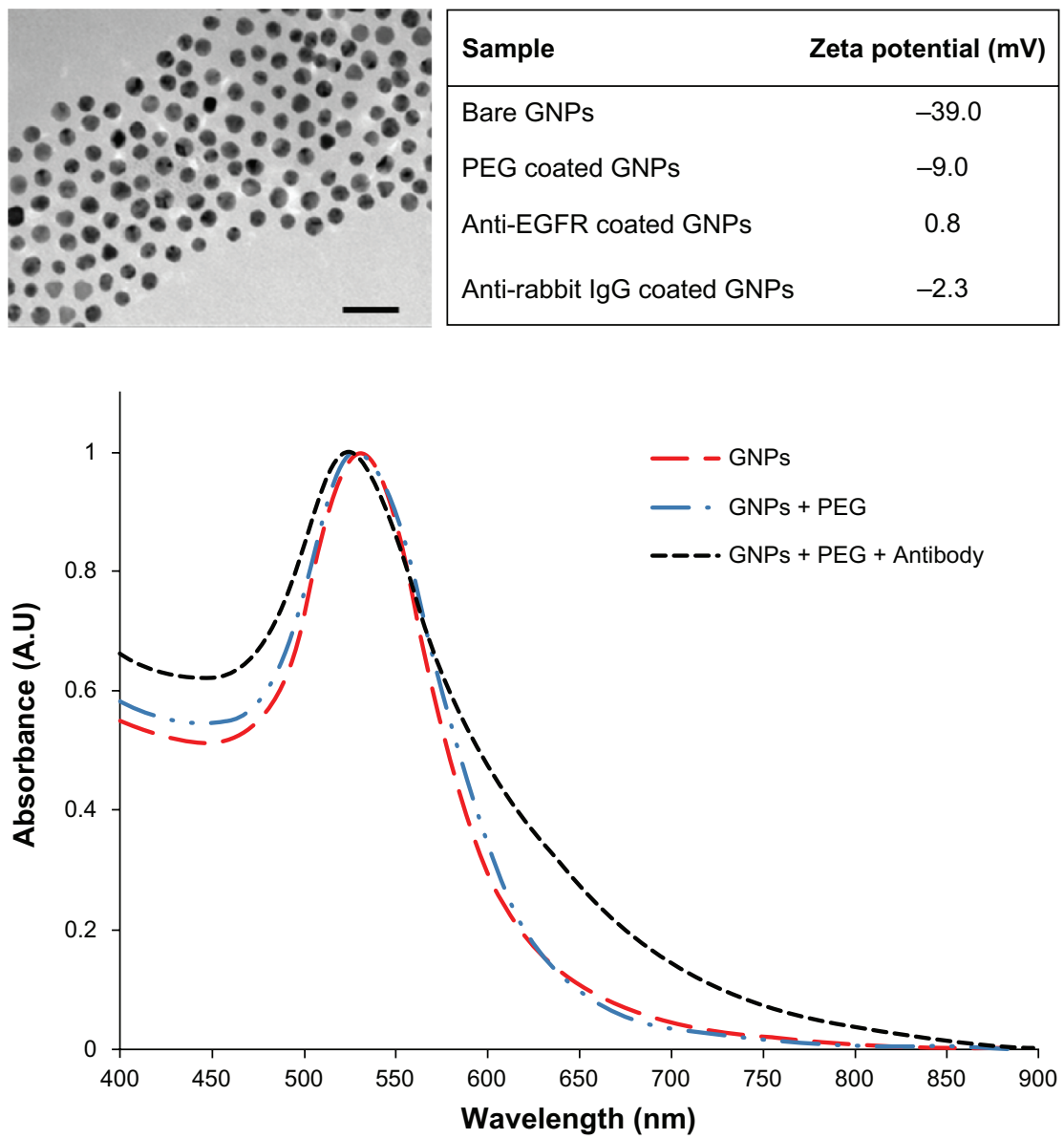

Figure I Characterization of gold nanoparticles (GNPs). Upper left: transmission electron microscopy image of $30 \mathrm{~nm}$ GNPs (scale bar I00 nm). Upper right: zeta potential measurements at the various stages of GNP coatings. Bottom: ultraviolet-visible spectroscopy of the bare GNPs, polyethylene glycol (PEG)ylated GNPs, and anti-epidermal growth factor receptor (EGFR)-coated GNPs.

Abbreviations: A.U, abitrary unit; IgG, immunoglobulin G.

by their maintenance of the same plasmon resonance. The coated GNPs were characterized using ultraviolet-visible spectroscopy (UV-1650 PC; Shimadzu Corporation, Kyoto, Japan) and zeta potential ${ }^{30}$ (ZetaSizer 3000HS; Malvern Instruments, Malvern, UK) (Figure 1).

\section{In vitro cell binding experiment}

A431 cells $\left(2.5 \times 10^{6}\right)$ in $5 \mathrm{~mL}$ Dulbecco's modified Eagle's medium containing $5 \%$ fetal calf serum, $0.5 \%$ penicillin, and $0.5 \%$ glutamine were divided into two groups for a quantitative cell binding study (each experimental group was run in triplicate). The first group was incubated with $50 \mu \mathrm{L}$ of anti-EGFR-coated GNPs $(25 \mathrm{mg} / \mathrm{mL})$ for 30 minutes at $37^{\circ} \mathrm{C}$ and the second group (negative control) was incubated at the exact same conditions with anti-rabbit IgG-coated GNPs. After incubation, the medium was washed twice with phosphate buffered saline followed by addition of $1 \mathrm{~mL}$ of aqua regia. After evaporation of the acid, the sediment was dissolved in $5 \mathrm{~mL} 0.05 \mathrm{M}$ hydrochloride.
The gold concentrations of the samples were quantified by atomic absorption spectroscopy (AA 140; Agilent Technologies, Santa Clara, CA).

\section{In vivo experiment}

A431 cells $\left(2 \times 10^{6}\right)$ were injected subcutaneously into the back flank area of six nude mice of age 10-11 weeks. The mice were divided into two groups of three each for active (GNPs coated with anti-EGFR antibodies) and passive (GNPs coated with anti-rabbit IgG antibodies) targeting studies. As a control, the two mice groups were scanned before GNPs injection.

When the tumor size reached a diameter of 4-5 $\mathrm{mm}$, the nude mice received $200 \mu \mathrm{L}$ ( $25 \mathrm{mg} / \mathrm{mL}$ gold) of immunotargeted GNPs by tail vein injection. The mice were scanned before injection to determine the $\mathrm{CT}$ baseline, as well as every hour post injection up to 6 hours. The scans were performed using a clinical 64 detector CT scanner (LightSpeed VCT, GE Healthcare, Little Chalfont, UK), with scanning parameters 
of $80 \mathrm{kVp}$ and $500 \mathrm{mAs}$. The contrast enhancement was quantitatively determined according to the differential contrast in CT numbers (in Hounsfield units [HU]), compared to that of the same mouse before injection. All CT scans were performed under appropriate anesthesia. Up to 7 days post intravenous injection, mouse behavior was normal and no evidence was observed of toxicity.

\section{Results and discussion In vitro cell binding experiment}

In order to evaluate the specificity of the interaction between the antibody-coated GNPs and the A431 SCC cancer cells (which highly express EGFR), two types of GNPs were introduced to the cells; the first was specifically coated with anti-EGFR antibody, whilst the second, which was used as a negative control, was coated with a nonspecific antibody (anti-rabbit IgG). Atomic absorption spectroscopy measurements quantitatively demonstrated that active tumor targeting (anti-EGFR-coated GNPs) was significantly more specific than the control experiment (antirabbit IgG-coated GNPs). The A431 cells took up $26.3 \pm 2.3 \mu \mathrm{g}$ of targeted GNPs $\left(3.9 \times 10^{4}\right.$ GNPs per A431 cell) whilst parallel cells in the negative control experiment absorbed only $0.2 \pm 0.01 \mu \mathrm{g}$ of GNPs $\left(3.4 \times 10^{3}\right.$ GNPs per cell). The results correlate well with previously published studies which report that head and neck SCC express from $2 \times 10^{4}$ to $2 \times 10^{6}$ EGFRs per cell. ${ }^{31,32}$

\section{In vivo CT experiments}

The ability of CT to detect tumors based on molecularlytargeted contrast agents was evaluated in mice bearing human head and neck tumors derived from the A431 SCC cell line. At the first stage of the experiment (0-3 hours), no significant difference was observed between the accumulation of the active and passive targeted particles. From that point on (3-6 hours), the accumulation of the actively targeted GNPs was steadily elevated, whilst the nontargeted particles were gradually cleared from the tumor. Maximal accumulation of the actively targeted particles was achieved 6 hours post GNPs injection. At this time point, the CT number of the tumor that was specifically targeted by the anti-EGFRcoated gold nanorods (Figure 2C) was over five times higher than the CT number of the same tumor in the same mouse before GNPs injection (190 $\pm 12 \mathrm{HU}$ versus $34 \pm 5 \mathrm{HU}$, respectively) (Figure $2 \mathrm{~A}$ ). This demonstrates that the GNPs were specifically attached to the SCC head and neck tumor at a high density, yielding a distinguishable contrast enhancement. The results also demonstrate that this small tumor (approximately 4-5 $\mathrm{mm}$ in diameter), which was undetectable without the GNPs contrast agents (Figure 2A),

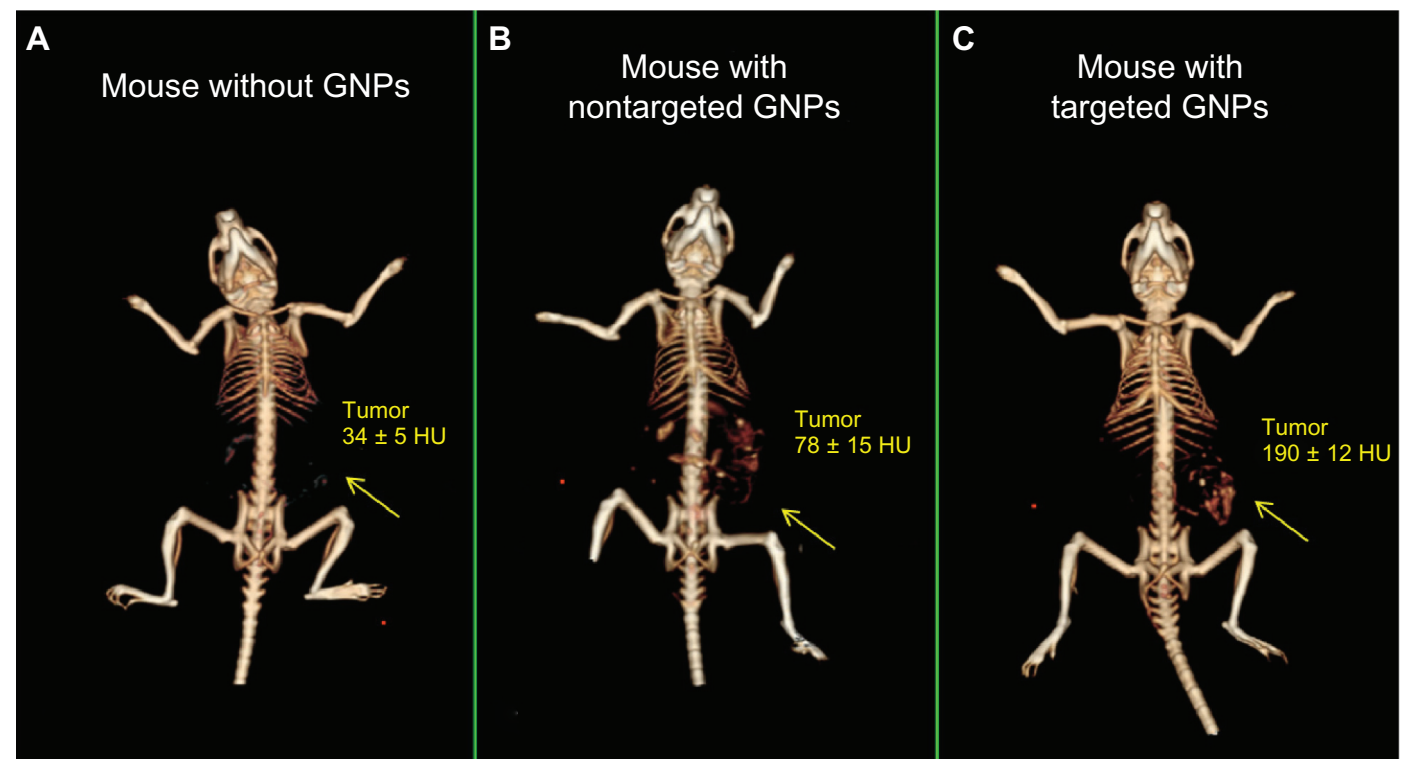

Figure 2 In vivo X-ray computed tomography (CT) volume-rendered images of (A) mouse before injection of gold nanoparticles (GNPs), (B) mouse 6 hours postinjection of nonspecific immunoglobulin G GNPs as a passive targeting experiment, and (C) mouse 6 hours postinjection of anti-epidermal growth factor receptor (EGFR)-coated GNPs that specifically targeted the squamous cell carcinoma head and neck tumor. The anti-EGFR-targeted GNPs show clear contrast enhancement of the tumor (C, yellow arrow), which was undetectable without the GNPs contrast agents (A, yellow arrow). CT numbers represent the average Hounsfield units (HU) of the whole tumor area. All scans were performed using a clinical CT at 80 kVp, 500 mAs, collimation $0.625 \times 64 \mathrm{~mm}$ and 0.521 pitch size (64 detector CT scanner, LightSpeed VCT; GE Healthcare, Little Chalfont, UK). 
was clearly visible and therefore can be easily detected after being specifically targeted by GNPs (Figure 2C).

Active and passive targeting mechanisms were compared at a single time point (6 hours post GNPs injection). As shown in Figure 2, active tumor targeting (Figure 2C) is more efficient and specific than passive targeting (Figure 2B), possibly because of elevated tumor uptake and retention forces. Passive tumor targeting takes advantage of the leaky nature of the tumor vasculature, allowing the GNPs to reach the tumor; however, GNPs are more rapidly cleared from the tumor and accumulate in the kidney, liver, and spleen (Figure 2B), according to their well-described clearance mechanism. ${ }^{29,33}$ The active targeting mechanism is highly important for $\mathrm{CT}$ contrast agents not only because of the specificity of the findings, but even more importantly, because the large amount of contrast material in the targeted site can overcome the inherently low sensitivity of CT to contrast agents.

Another important parameter that should be noted is the high differential contrasts that were obtained in the above experiments. The signal (defined as the CT number of the organ post GNPs injection) relative to the background (defined as the CT number of the organ before GNPs injection) in the active study was significantly higher than the equivalent value obtained in the passive study. This results in poor contrast and low detectability of the passively targeted tumor (Figure 3). The lower accumulation of the passively targeted GNPs onto the tumor in comparison to the accumulation obtained in the actively targeted GNPs ( $78 \mathrm{HU}$ versus $190 \mathrm{HU}$, respectively), yields higher accumulation in the kidney (42 $\mathrm{HU}$ versus $34 \mathrm{HU}$, respectively) and liver (96 HU versus $63 \mathrm{HU}$, respectively).

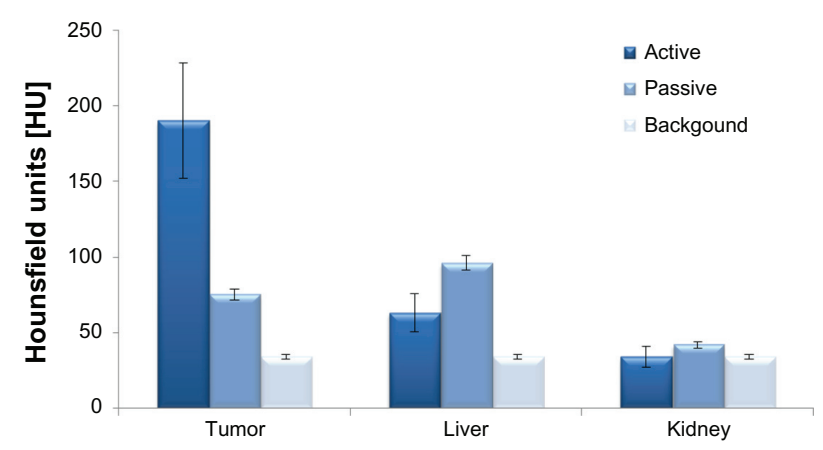

Figure 3 Biodistribution computed tomography results of anti-epidermal growth factor receptor-coated gold nanoparticles (active) and anti-immunoglobulin G-coated gold nanoparticles (passive) in the tumor, liver, and kidney (6 hours post intravenous injection). Background is defined as the computed tomography number of the organ before injection of gold nanoparticles. Each group was comprised of three mice.
It is important to note that these results were obtained using a clinical $\mathrm{CT}$, which has a relatively low resolution compared to a micro CT; therefore, they yield high potential for clinical application.

\section{Conclusion}

In summary, this study demonstrated the feasibility of molecular cancer imaging with clinical CT. Targeted GNPs are capable of producing contrast enhancement (increased X-ray attenuation) so that CT can detect cancer based on molecular markers rather than on anatomical structures. The strong selective X-ray attenuation by gold nanoprobe assembly is distinct from the attenuation of other cell types and tissues, and therefore transforms the targeted cancer into highly distinct features that are easy to diagnose in $\mathrm{CT}$ imaging. It was further shown that the CT number of the molecularly-targeted tumor is over five times higher than the corresponding number of an identical but untargeted tumor and that active tumor targeting is more efficient and specific than passive targeting. This targeted molecular imaging tool is expected to provide a new method to investigate in vivo the expression and activity of cancer-related biomarkers, to study tumor physiology, and to trace tumors' molecular processes. This primary research will pave the way for a further future study to characterize important parameters, such as optimal dosing and detection limit, ie, the smallest tumor size that can be detected.

\section{Acknowledgments}

This work was supported by a Research and Development - Chief Scientist Nofar grant, Merck Sorono, the Elias, Genevieve, and Geogianna Atol Charitable Trust, and the Katz Family Grant Incentive Program. We would like to acknowledge Professor Yossef Yarden for his assistance.

\section{Disclosure}

The authors report no conflicts of interest in this work.

\section{References}

1. Branstetter BF 4th, Blodgett TM, Zimmer LA, et al. Head and neck malignancy: is PET/CT more accurate than PET or CT alone? Radiology. 2005;235(2):580-586.

2. Ross B, Rehemtulla A, Koo YEL, et al. Photonic and magnetic nanoexplorers for biomedical use: from subcellular imaging to cancer diagnostics and therapy. Proceedings of the SPIE - The International Society for Optical Engineering; June 21-25, 2004; Glasgow, Scotland. Bellingham, WA: International Society for Optical Engineering; 2004:76-83.

3. Kopelman R, Koo YEL, Philbert M, et al. Multifunctional nanoparticle platforms for in vivo MRI enhancement and photodynamic therapy of a rat brain cancer. J Magn Magn Mater. 2005;293(1):404-410. 
4. Peng C, Wang H, Guo R, et al. Acetylation of dendrimer-entrapped gold nanoparticles: synthesis, stability, and x-ray attenuation properties. J Appl Polym Sci. 2011;119(3):1673-1682.

5. Hainfeld JF, Slatkin DN, Focella TM, Smilowitz HM. Gold nanoparticles: a new X-ray contrast agent. Br J Radiol. 2006;79(939):248-253.

6. Kim D, Park S, Lee JH, Jeong YY, Jon S. Antibiofouling polymercoated gold nanoparticles as a contrast agent for in vivo $\mathrm{X}$-ray computed tomography imaging. J Am Chem Soc. 2007;129(24):7661-7665.

7. Cai QY, Kim SH, Choi KS, et al. Colloidal gold nanoparticles as a blood-pool contrast agent for $\mathrm{x}$-ray computed tomography in mice. Invest Radiol. 2007;42(12):797-806.

8. Xiao M, Nyagilo J, Arora V, et al. Gold nanotags for combined multi-colored Raman spectroscopy and x-ray computed tomography. Nanotechnology. 2010;21(3):035101.

9. De Vries A, Custers E, Lub J, van den Bosch S, Nicolay K, Grull H. Block-copolymer-stabilized iodinated emulsions for use as CT contrast agents. Biomaterials. 2010;31(25):6537-6544.

10. Bonitatibus PJ Jr, Torres AS, Goddard GD, FitzGerald PF, Kulkarni AM. Synthesis, characterization, and computed tomography imaging of a tantalum oxide nanoparticle imaging agent. Chem Comm (Camb). 2010; 46(47):8956-8958.

11. Kim D, Yu MK, Lee TS, Park JJ, Jeong YY, Jon S. Amphiphilic polymer-coated hybrid nanoparticles as CT/MRI dual contrast agents. Nanotechnology. 2011;22(15):155101.

12. Rabin O, Manuel Perez J, Grimm J, Wojtkiewicz G, Weissleder R. An $\mathrm{X}$-ray computed tomography imaging agent based on long-circulating bismuth sulphide nanoparticles. Nat Mater. 2006;5(2):118-122.

13. Wang $\mathrm{H}$, Zheng LF, Peng $\mathrm{C}$, et al. Computed tomography imaging of cancer cells using acetylated dendrimer-entrapped gold nanoparticles. Biomaterials. 2011;32(11):2979-2988.

14. Noguchi Y, Wu J, Duncan R, et al. Early phase tumor accumulation of macromolecules: a great difference in clearance rate between tumor and normal tissues. Jpn J Cancer Res. 1998;89(3):307-314.

15. Dvorak HF, Nagy JA, Dvorak JT, Dvorak AM. Identification and characterization of the blood-vessels of solid tumors that are leaky to circulating macromolecules. Am J Pathol. 1988;133(1):95-109.

16. Popovtzer R, Agrawal A, Kotov NA, et al. Targeted gold nanoparticles enable molecular CT imaging of cancer. Nano Lett. 2008;8(12): 4593-4596.

17. Hainfeld JF, O’Connor MJ, Dilmanian FA, Slatkin DN, Adams DJ, Smilowitz HM. Micro-CT enables microlocalisation and quantification of Her2-targeted gold nanoparticles within tumour regions. Br J Radiol. 2011;84(1002):526-533.

18. Chanda N, Kattumuri V, Shukla R, et al. Bombesin functionalized gold nanoparticles show in vitro and in vivo cancer receptor specificity. Proc Natl Acad Sci U S A. 2010;107(19):8760-8765.
19. Perrault SD, Walkey C, Jennings T, Fischer HC, Chan WC. Mediating tumor targeting efficiency of nanoparticles through design. Nano Lett. 2009;9(5):1909-1915.

20. Longmire M, Choyke PL, Kobayashi H. Clearance properties of nanosized particles and molecules as imaging agents: considerations and caveats. Nanomedicine (Lond). 2008;3(5):703-717.

21. Zhang G, Yang Z, Lu W, et al. Influence of anchoring ligands and particle size on the colloidal stability and in vivo biodistribution of polyethylene glycol-coated gold nanoparticles in tumor-xenografted mice. Biomaterials. 2009;30(10):1928-1936.

22. Haigler H, Ash JF, Singer SJ, Cohen S. Visualization by fluorescence of binding and internalization of epidermal growth-factor in human carcinoma cells A-431. Proc Natl Acad Sci U S A. 1978;75(7): 3317-3321

23. Grandis JR, Melhem MF, Gooding WE, et al. Levels of TGF-alpha and EGFR protein in head and neck squamous cell carcinoma and patient survival. J Natl Cancer Inst. 1998;90(11):824-832.

24. Baselga J. The EGFR as a target for anticancer therapy - focus on cetuximab. Eur J Cancer. 2001;37 Suppl 4:S16-S22.

25. Gindy ME, Prud'homme RK. Multifunctional nanoparticles for imaging, delivery and targeting in cancer therapy. Expert Opin Drug Deliv. 2009; 6(8):865-878

26. Enüstun BV, Turkevich J. Coagulation of colloidal gold. J Am Chem Soc. 1963;85(21):3317-3328.

27. Moghimi SM, Hunter AC, Murray JC. Long-circulating and targetspecific nanoparticles: theory to practice. Pharmacol Rev. 2001;53(2): 283-318.

28. Qian X, Peng XH, Ansari DO, et al. In vivo tumor targeting and spectroscopic detection with surface-enhanced Raman nanoparticle tags. Nat Biotechnol. 2008;26(1):83-90.

29. Wang X, Qian X, Beitler JJ, et al. Detection of circulating tumor cells in human peripheral blood using surface-enhanced raman scattering nanoparticles. Cancer Res. 2011;71(5):1526-1532.

30. Maldiney T, Richard C, Seguin J, Wattier N, Bessodes M, Scherman D. Effect of core diameter, surface coating, and PEG chain length on the biodistribution of persistent luminescence nanoparticles in mice. ACS Nano. 2011;5(2):854-862.

31. Todd R, Wong DT. Epidermal growth factor receptor (EGFR) biology and human oral cancer. Histol Histopathol. 1999;14(2):491-500.

32. Stanton P, Richards S, Reeves J, et al. Epidermal growth-factor receptor expression by human squamous-cell carcinomas of the head and neck, cell-lines and xenografts. Br J Cancer. 1994;70(3):427-433.

33. Li SD, Huang L. Pharmacokinetics and biodistribution of nanoparticles. Mol Pharm. 2008;5(4):496-504.
International Journal of Nanomedicine

\section{Publish your work in this journal}

The International Journal of Nanomedicine is an international, peerreviewed journal focusing on the application of nanotechnology in diagnostics, therapeutics, and drug delivery systems throughout the biomedical field. This journal is indexed on PubMed Central, MedLine, CAS, SciSearch $\AA$, Current Contents ${ }^{\circledR} /$ Clinical Medicine,

\section{Dovepress}

Journal Citation Reports/Science Edition, EMBase, Scopus and the Elsevier Bibliographic databases. The manuscript management system is completely online and includes a very quick and fair peer-review system, which is all easy to use. Visit http://www.dovepress.com/ testimonials.php to read real quotes from published authors. 